\title{
Wesentliche Änderungen im Strahlenschutzrecht ab 2019
}

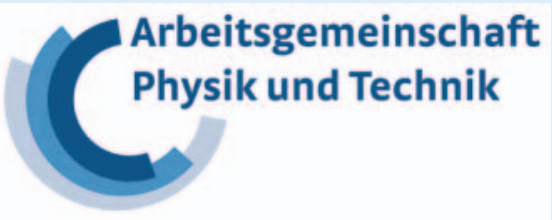

Das Jahr 2019 ist mit einer kompletten Novellierung des Strahlenschutzrechtes gestartet. Inhaltlich ist zwar vieles beim Alten geblieben aber es verteilt sich nun auf zwei Werke (Strahlenschutzgesetz und Strahlenschutzverordnung). Zudem wurde die Trennung von Röntgendiagnostik sowie Strahlentherapie und Nuklearmedizin in zwei verschiedenen Verordnungen aufgehoben. Die wichtigsten Änderungen werden in diesem Beitrag kurz zusammengefasst.

Umfassende Informationen bietet zudem das „Forum Strahlenschutzrecht“ (früher Forum Röntgenverordnung) auf dem diesjährigen Deutschen Röntgenkongress.

\section{Medizinphysik-Experte wird für viele Neuanlagen Pflicht}

Ab 2019 verlangt der Gesetzgeber bereits bei der Anzeige eines neuen CT's oder einer Interventionsanlage einen Nachweis über Zusammenarbeit mit einem Medizinphysik-Experten (MPE). Hiervon betroffen ist auch jeder Betreiberwechsel.

Für alle anderen Anlagen wie Aufnahmeplätze oder diagnostische Durchleuchtungsanlagen ist dies nicht notwendig. Keinen Medizinphysik-Experten benötigen zudem derzeit alle Anlage, die vor dem 31.12.2018 in Betrieb gegangen sind. Diese „Alt“-Anlagen müssen erst ab dem 31.12.2022 durch einen MPE betreut werden (s. auch $\S 14$ und $\S 19$ StrISchG sowie $\S 131$ StrSchV).

Präzise fordert das Strahlenschutzgesetz die Hinzuziehung des MPE zur Mitarbeit. Dies bedeutet dass er nicht bei allen Maßnahmen anwesend sein muss und damit auch als externer Dienstleister fungieren kann.

Der MPE muss über die Fachkunde für Medizinphysikexperten nach der Richtlinie „Fachkunde und Kenntnisse im Strahlen- schutz bei dem Betrieb von Röntgeneinrichtungen in der Medizin oder Zahnmedizin“ vom 22. Dezember 2005 verfügen. MPE aus anderen Fachgebieten (Strahlentherapie und Nuklearmedizin) sind nicht automatisch fachkundig im Sinne dieser Richtlinie. Eine Regelung über notwendige zusätzliche Qualifikationsmaßnahmen für diese Kollegen ist bei den zuständigen Ministerien in Arbeit.

Für Deutschland muss mit etwa 500 Neuanlagen pro Jahr gerechnet werden. Da der Betreuungsaufwand zur Optimierung und Überwachung dieser Anlagen lediglich mehrere Arbeitstage pro Jahr erfordert, liegt der Bedarf in den nächsten vier Jahren bei etwa 50 zusätzlichen MPE pro Jahr. Engpässe sind somit vorprogrammiert.

Um gerade in den ersten Monaten kritische Engpässe zu vermeiden, hat sich die APT entschlossen, eine Liste von erfahrenen MPE zu veröffentlichen. Die APT kann und darf aber in diesem Zusammenhang aber nicht als Vermittler auftreten. Der Kontakt kann also nur durch direkte Ansprache erfolgen.

Zusätzlich haben APT und DGMP den Ländern ein Mentorenprogramm vorgeschlagen, mit dem die Versorgungslage qualifizierter MPE innerhalb eines angemessenen Zeitraums sichergestellt werden kann. Die Gespräche mit den Ministerien zur Finanzierung eines solchen Programms laufen. Eine Entscheidung steht aber noch aus.

\section{Weitere Informationen:}

\section{SSK-Empfehlung}

Hinzuziehung eines Medizinphysik-Experten bei medizinisch-radiologischen Tätigkeiten Umsetzung der Anforderungen der Richtlinie 2013/59/Euratom; September 2017

$\S 14$ Abs. 1 Nr. 2b StrlSchG

$\S 19$ Abs. 3 Nr. 7 StrlSchG

$\S 131$ Absatz 2 StrSchV

...(2) Der Strahlenschutzverantwortliche hat dafür zu sorgen, dass ein Medizinphysik-Experte zur Mitarbeit hinzugezogen wird bei ...

...3. Untersuchungen mit ionisierender Strahlung, die mit einem Computertomografen oder mit Geräten zur dreidimensionalen Bildgebung von Objekten mit niedrigem
Röntgenkontrast durchgeführt werden mit Ausnahme der Tomosynthese, und

4. Interventionen, bei denen die Röntgeneinrichtungen zur Durchleuchtung eingesetzt werden.

Die Aufgaben des MPE sind in der StrSchV $\S 132$ definiert.

\section{Frist für die Anzeige eines Neugerätes auf 4 Wochen verlängert}

Nach der Röntgenverordnung musste jedes Gerät 2 Wochen vor Inbetriebnahme angezeigt werden. In den vielen Fällen waren die Aufsichtsbehörden sehr entgegenkommend und haben lediglich verlangt, dass Datenblätter und Strahlenschutzplan zu diesem Zeitpunkt vorliegen mussten. Der Sachverständigenbericht konnte dann am Tage vor der eigentlichen Inbetriebnahme eingereicht werden. Damit kam es nicht zu einem Stillstand einer betriebsbereiten Anlage. Ob dies nach der neuen Regelung mit deutlich verlängerter Anzeigefrist noch ähnlich abläuft sollte jeder Betreiber frühzeitig mit seiner Behörde abklären.

\section{Meldepflicht für bedeutsame Vorkommnisse}

Ebenfalls ab 2019 sind die Strahlenschutzverantwortlichen verpflichtet jedes „bedeutsame Vorkommnis“ zu analysieren, Maßnahmen zur Vermeidung zu ergreifen und eine Meldung an die Aufsichtsbehörde vorzunehmen.

Die Umsetzung setzt voraus, dass bedeutsame Vorkommnisse im Betrieb erkannt und registriert werden. Ein bedeutsames Ereignis ist dabei laut Begriffsbestimmung $\S 1$ StrSchV:

„(22) Vorkommnis: Ereignis in einer geplanten Expositionssituation, das zu einer unbeabsichtigten Exposition geführt hat, geführt haben könnte oder führen könnte. Kein Vorkommnis liegt vor, wenn das Ereignis für den Strahlenschutz nicht relevant ist." 
Als bedeutsame Vorkommnisse werden nach § 108 StrlSchV und deren Anlagen entweder Dosisüberschreitungen nach einem komplexen Kriterienkatalog oder

- Jede Wiederholung einer Anwendung, insbesondere aufgrund einer Körperteilverwechslung, eines Einstellungsfehlers oder eines vorausgegangenen Gerätedefekts, wenn es zu einer erheblichen zusätzlichen Exposition (s. u.) gekommen ist.

- Jede Personenverwechslung, wenn es zu einer erheblichen zusätzlichen Exposition (s. u.) gekommen ist.

- Jedes Auftreten einer deterministischen Wirkung, die für die festgelegte Untersuchung nicht zu erwarten war.

Bei den Dosisüberschreitungen wird grundsätzlich zwischen der Exposition einer Einzelperson oder einer Gruppe unterschieden. Letzteres führt dazu, dass auch permanente, leichtere Dosiserhöhungen z. B. durch ungünstige Protokolle auffällig werden und gemeldet werden müssen. Die Gruppenbetrachtung wird nur nötig, wenn die Aktionsschwelle von $200 \%$ Überschreitung des DRW (=3× DRW) erreicht ist. Danach erfolgt eine Bewertung der letzten 20 Untersuchungen gleichen Typs am gleichen Gerät. Liegt der Mittelwert dieser Untersuchungen beim 2-fachen des DRW ist die Meldeschwelle erreicht (siehe Abbildung).

\section{Beispiel: CT Thorax (bis einschließlich Nebennieren)}

Trigger ist die Überschreitung des DRW um $200 \%$ für eine einzelne Untersuchung. Für einen CT-Thorax liegt der Referenzwert für das Dosislängenprodukt bei $350 \mathrm{mGy}^{*} \mathrm{~cm}$. Bei dem Einmaligen Auftauchen eines Dosiswertes von mehr als 1050 mGy* cm für das Dosislängenprodukt wäre also die Aktionsschwelle erreicht.

Dann müssen die letzten 20 Untersuchungen des gleichen Bereichs/Protokolls vor dem Ereignis geprüft werden und der Mittelwert über diese 20 Untersuchungen gebildet werden.
Meldepflicht bei „bedeutsamen Vorkommnissen“ in der Röntgendiagnostik Strahlenschutzverordnung $\$ 108$

\begin{tabular}{|c|c|c|c|c|}
\hline Bezug & Maßnahmentyp & Schwellenart & Wert & Häufigkeit \\
\hline \multirow[t]{2}{*}{ Gruppe } & \multirow{2}{*}{$\begin{array}{l}\text { Computer- } \\
\text { tomographie, } \\
\text { Durchleuchtung, } \\
\text { Intervention }\end{array}$} & Aktionsschwelle & $3 \times$ DRW & $\begin{array}{l}\text { einmalig, danach } \\
\text { Prüfung der } \\
\text { Meldeschwelle }\end{array}$ \\
\hline & & Meldeschwelle & $2 \times$ DRW & $\begin{array}{l}\text { Mittelwert der } \\
\text { letzten } 20 \\
\text { Untersuchungen }\end{array}$ \\
\hline \multirow{4}{*}{ Person } & $\begin{array}{l}\text { Computer- } \\
\text { tomographie }\end{array}$ & Meldeschwelle & $\begin{array}{l}\mathrm{CTDI}_{\text {Him }}>120 \mathrm{mGy} \\
\mathrm{CTDI}_{\text {Körper }}>80 \mathrm{mGy}\end{array}$ & einmalig \\
\hline & $\begin{array}{l}\text { diagn. } \\
\text { Durchleuchtung }\end{array}$ & Meldeschwelle & DFP $>20.000 \mathrm{cGy}^{*} \mathrm{~cm}^{2}$ & einmalig \\
\hline & \multirow[t]{2}{*}{ Intervention } & Aktionsschwelle & DFP $>50.000 \mathrm{cGy}^{*} \mathrm{~cm}^{2}$ & $\begin{array}{l}\text { einmalig, danach } \\
\text { Prüfung der } \\
\text { Meldeschwelle }\end{array}$ \\
\hline & & Meldeschwelle & $\begin{array}{l}\text { deterministischer Schaden } \\
\text { innerhalb von } 21 \text { Tagen }\end{array}$ & einmalig \\
\hline \multicolumn{3}{|c|}{ H. Lenzen, Münster - nach StriSchV ş108 } & $1 \mathrm{cGy}^{*} \mathrm{~cm}^{2}=1 \mu \mathrm{Gy}^{*} \mathrm{~m}^{2}$ & \\
\hline
\end{tabular}

Überschreitet dieser den DRW um $100 \%$ ist der Vorgang ein meldepflichtiges Ereignis.

Ist für das obige Beispiel also der Mittelwert der letzten 20 CT-Thorax-Untersuchungen höher als $700 \mathrm{mGy}^{*} \mathrm{~cm}$ so wäre der Vorgang meldepflichtig im Sinne der StrSchV.

Das Konzept ist in vielen Punkten problematisch. So bleiben die Scanlänge und die Strahlenempfindlichkeit der Organe und Gewebe unberücksichtigt. Probleme könnten auch bei dynamischen CT-Untersuchungen wie z. B. der Hirnperfusion auftreten. Hier besteht noch Anpassungs- und Klärungsbedarf im Verordnungstext. Zudem führt die Meldeschwelle bei Interventionen dazu, dass jeder Patient mit über 50000 cGy* $^{*} \mathrm{~cm}^{2}$ nach 21 Tagen auf deterministische Schäden hin untersucht werden muss.

Die Überwachung und Dokumentation der obigen Kriterien kann durch den Einsatz eines Dosismanagementsystems (DMS) deutlich vereinfacht werden. Aber diese Systeme führen auch zu einem erhöhten Betreuungsaufwand durch fachkundiges Personal. Auf Knopfdruck geht das nicht.

\section{Grenzwert für die Linsendosis stark abgesenkt}

Der Grenzwert für die Linsendosis wurde von $150 \mathrm{mSv}$ auf $20 \mathrm{mSv}$ pro Jahr sehr deutlich abgesenkt. Problematisch wird dies nur bei Interventionen. Hier lässt sich der Wert aber durch den richtigen Einsatz patientennaher Schutzmaßnahmen in Verbindung einhalten. Die Messstellen, die bisher Prüfplaketten und Fingerringdosimeter geliefert haben, stellen auch Linsendosimeter bereit. Hilfreich können hier auch Echtzeitdosimeter sein, die die Exposition während der gesamten Untersuchung überwachen und anzeigen.

\section{Röntgenpass fällt weg}

Der Röntgenpass diente in den letzten Jahren dazu, den Arzt auf bereits erfolgte Voruntersuchungen hinzuweisen. Dieses Vorgehen wird im neuen Strahlenschutzrecht nicht mehr erwähnt. Es ist daher aus rechtlichen Gründen nicht mehr notwendig einen solchen Pass auszufüllen oder auszuhändigen. Trotzdem werden viele Patienten auch weiterhin den Wunsch verspüren, einen solchen Pass zu führen. Hier wird man als Klinik oder Praxis entscheiden müssen, wie man damit umgeht. mit einer geeigneten Strahlenschutzbrille 


\section{Überschreitungen} von Diagnostischen Referenzwerten (DRW) müssen begründet werden

Nach § 85 StrISchG sind alle Überschreitungen eines DRW zu begründen. Die bedeutet für die Dokumentation einen erheblichen Mehraufwand. Pro Jahr wird es in jeder Abteilung zu mehreren Tausend Überschreitungen kommen. Diese waren bisher auch unproblematisch solange der Mittelwert aller Untersuchungen dieses Typs unterhalb des DRW lag. Zudem sind die meisten RIS-Systeme zumindest jetzt noch nicht auf dieses Pflichtfeld eingerichtet.

Da der DRW aber nicht für einzelne Untersuchungen definiert ist, sondern über eine größere Gesamtheit, ist aus unserer Sicht nicht jede einzelne, sondern die dauerhafte Überschreitung des DRW zu dokumentieren. Dies wird inzwischen auch vom BMU und den Ärztlichen Stellen so gesehen. Leider liegt aber derzeit noch keine rechtsverbindliche Klarstellung des BMU vor.

\section{Personendosimetrie nur noch mit zentraler}

\section{Kennummer}

Nach $§ 170$ StrISchG benötigen alle beruflich exponierte Personen, für die Eintragungen ins Strahlenschutzregister des BfS ab dem 31.12.2018 eine eindeutige persönliche Kennnummer: die Strahlenschutzregisternummer (SSR-Nummer).
Die SSR-Nummer erleichtert und verbessert die Zuordnung und Bilanzierung der individuellen Dosiswerte aus der beruflichen Strahlenexposition im Strahlenschutzregister. Ohne SSR gibt es keine Plaketten für die Personendosimetrie.

Die SSR-Nummer wird vom BfS vergeben. Sie wird durch eine nicht rückführbare Verschlüsselung aus der Sozialversicherungsnummer (§ 147 SGB VI) und den Perso- nendaten des zu überwachenden Beschäftigten abgeleitet.

Jede Institution kann die SSR durch hochladen einer Excel Tabelle mit der Mitarbeiterdaten über die Internetseite des BfS generieren. Auch Einzelpersonen können auf diesem Wege eine Nummer generieren.

H. Lenzen; G. Stamm, 03.2019 